\title{
Optimal Disturbances Rejection Control for Autonomous Underwater Vehicles in Shallow Water Environment
}

\author{
Qing Yang, ${ }^{1}$ Hao Su, ${ }^{1}$ Gong-You Tang, ${ }^{1}$ and De-Xin Gao ${ }^{2}$ \\ ${ }^{1}$ College of Information Science and Engineering, Ocean University of China, Qingdao 266100, China \\ ${ }^{2}$ College of Automation and Electronic Engineering, Qingdao University of Science \& Technology, Qingdao 266042, China
}

Correspondence should be addressed to Gong-You Tang; gtang@ouc.edu.cn

Received 28 April 2016; Accepted 26 January 2017; Published 29 March 2017

Academic Editor: Huaguang Zhang

Copyright (C) 2017 Qing Yang et al. This is an open access article distributed under the Creative Commons Attribution License, which permits unrestricted use, distribution, and reproduction in any medium, provided the original work is properly cited.

To deal with the disturbances of wave and current in the heading control of Autonomous Underwater Vehicles (AUVs), an optimal disturbances rejection control (ODRC) approach for AUVs in shallow water environment is designed to realize this application. Based on the quadratic optimal control theory, the AUVs heading control problem can be expressed as a coupled two-point boundary value (TPBV) problem. Using a recently developed successive approximation approach, the coupled TPBV problem is transformed into solving a decoupled linear state equation sequence and a linear adjoint equation sequence. By iteratively solving the two equation sequences, the approximate ODRC law is obtained. A Luenberger observer is constructed to estimate wave disturbances. Simulation is provided to demonstrate the effectiveness of the presented approach.

\section{Introduction}

Nowadays, there has been increasing interest in the use of AUVs to expand the ability to survey ocean areas, for example, exploration and exploitation of seafloor minerals, oceanographic mapping, and underwater pipelines tracking [1]. However, due to highly nonlinear and strongly coupled dynamics of AUVs and the environmental disturbances (such as currents and waves), it is always a challenge to design controller for AUVs. Recently, numerous nonlinear control methods have been utilized to achieve improved performances for motion control of AUVs. Typical results include sliding control, adaptive control, and optimal control. In [2-4], the sliding mode control techniques were applied to motion control of AUVs. In [5-7], the backstepping control approach was employed to design controller for path following of underactuated AUVs. In $[8,9], \mathrm{H}_{2}$ and $H_{\infty}$ controllers were presented for motion control of AUVs. In [10-12], the adaptive control methods were utilized for motion control of AUVs to improve robustness of the control systems. In [13], a suboptimal control approach was proposed for motion control of AUVs. In [14], a feedback linearization technique was applied for AUVs tracking problem. In [15], the combined problem of trajectory planning and tracking control for underactuated AUVs in the horizontal plane was addressed, and a backstepping approach was presented. In [16], Lyapunov approach and backstepping control approach were applied to design path-following controller of an AUV in the horizontal plane with constant ocean currents. In [17], an $H_{\infty}$ robust fault-tolerant controller was designed to improve the security and reliability of navigation and enhance the accuracy and robustness of navigation control system for AUVs.

Note that nonlinearity and disturbances such as currents and wave are unavoidable in AUVs control systems, which affect the performance of the AUVs system. At present, there are many methods to deal with these problems. In [18], an output feedback control approach was proposed for the trajectory tracking control of AUVs, which moved in shallow water areas. In [19], for the problem of dynamic positioning and way-point tracking of underactuated AUVs in the presence of constant unknown ocean currents and parametric modeling uncertainty, a nonlinear adaptive controller was proposed. In [20], a stable sliding mode controller was designed, which can track AUVs along the desired trajectory in complex sea conditions. In [21], an adaptive 


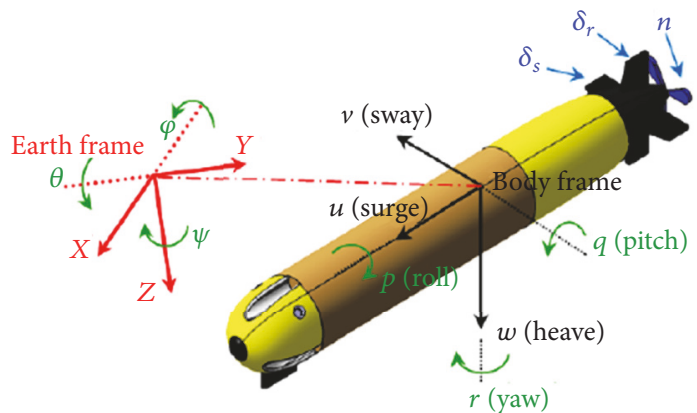

Figure 1: A schematic of the six-DOF AUVs model.

output feedback controller was presented for AUVs named ODIN to track a desired trajectory with bounded errors in wave disturbances condition. In [22], a second-order sliding mode controller was proposed for an AUV, which can compensate for disturbances such as waves, currents, and buoyancy force.

In the field of modern control, one of the key ideas is the use of optimization and optimal control theory to give a systematic procedure for the design of feedback control systems [23-25], LQR approach provides one of the most useful techniques for designing state feedback controllers. In order to overcome disturbances and nonlinearities for the AUV system, in this paper, an ODRC approach is proposed based on the quadratic optimal control theory. Firstly, the AUVs model and wave disturbances model are obtained, and according to disturbances type, an integral unit is introduced to eliminate its ocean current disturbances effect, and a feedforward control is used to reject wave disturbances. Secondly, for the AUVs heading control system, the coupled TPBV problem is derived from the maximum principle of optimal control theory. Then by using a successive approximation approach $[26,27]$, the coupled TPBV problem is transformed into solving two decoupled linear differential sequences in state vectors and adjoint vectors. By iterative solution, the ODRC law is obtained, and a Luenberger disturbances observer is constructed to make it realizable. The contribution in this paper is the ODRC approach which is applied to design rejection controller for AUVs in shallow water environment, which only requires solving the Riccati equation and the Sylvester matrix equation one time, while mainly solving a recursion formula of adjoint vectors.

This paper is organized as follows. In Section 2, the model of AUVs motion in horizontal plane is introduced, and the system of shallow wave disturbances is constructed. Section 3 presented an ODRC design for AUVs. Simulation validates the effectiveness of the designed controller under wave disturbances in Section 4. Section 5 provides the concluding remarks.

\section{System Models}

2.1. Mathematical Model of AUVs. A schematic of the sixDOF AUVs model with related coordinate system is shown in Figure 1.
The two reference frames are applied to the model: Earth-fixed frame and body-fixed frame. Descriptions of the parameters are expressed in Table 1 [28-30].

The Earth-fixed frame is treated as an inertial frame. In order to facilitate the analysis and synthesis for AUVs, the coupling effect between the roll surface movement and two cases of plane motion is usually ignored, and then the vehicle motion is divided into horizontal and vertical movement. The heading motion equations in horizontal plane are given in dimensional form as

$$
\begin{aligned}
m[\dot{v}+u r-w p] & =\sum Y \\
I_{z} \dot{r}+\left(I_{y}-I_{x}\right) p q & =\sum N \\
\dot{\psi} & =\frac{(q \sin \phi+r \cos \phi)}{\cos \theta},
\end{aligned}
$$

where

$$
\begin{aligned}
& \sum Y=\frac{1}{2} \rho L^{4}\left[Y_{\dot{r}}^{\prime} \dot{r}+Y_{\dot{p}}^{\prime} \dot{p}+Y_{p q}^{\prime} p q+Y_{r|r|}^{\prime} r|r|\right]+\frac{1}{2} \\
& \cdot \rho L^{3}\left[Y_{\dot{v}}^{\prime} \dot{v}+Y_{u r}^{\prime} u r+Y_{w p}^{\prime} w p\right. \\
& \left.+Y_{v|r|}^{\prime} \frac{v}{|v|} \sqrt{v^{2}+w^{2}}|r|\right]+\frac{1}{2} \rho L^{2}\left[Y_{u v}^{\prime} u v\right. \\
& \left.+Y_{v|v|}^{\prime} v \sqrt{v^{2}+w^{2}}\right]+(W-B) \cos \theta \sin \phi+\frac{1}{2} \\
& \cdot \rho L^{2} Y_{\delta_{r}}^{\prime} u^{2} \delta_{r}+d \\
& \sum N=\frac{1}{2} \rho L^{5}\left[N_{\dot{r}}^{\prime} \dot{r}+N_{p q}^{\prime} p q+N_{r|r|}^{\prime} r|r|\right]+\frac{1}{2} \rho L^{4}\left[N_{\dot{v}}^{\prime} \dot{v}\right. \\
& \left.\quad+N_{u r}^{\prime} u r+N_{w p}^{\prime} w p+N_{v q}^{\prime} v q+N_{|v| r}^{\prime} \sqrt{v^{2}+w^{2} r}\right]+\frac{1}{2} \\
& \cdot \rho L^{3}\left[N_{u v}^{\prime} u v+N_{v|v|}^{\prime} v \sqrt{v^{2}+w^{2}}\right]+\frac{1}{2} \rho L^{3} N_{\delta_{r}}^{\prime} u^{2} \delta_{r} \\
& \quad+d .
\end{aligned}
$$

In system (1), $\delta_{r}$ is the control rudder angle; $L, m, W$ and $B$, respectively, are the vehicle's length, quality, weight, and buoyancy; $\rho$ is the density of seawater; $I_{x}, I_{y}$, and $I_{z}$, respectively, are the vehicle's moment of inertia about $x_{-}, y_{-}$, and $z$-axis; $d$ is the external disturbances; $Y^{\prime}(\cdot)$ and $N^{\prime}(\cdot)$ are hydrodynamic coefficients.

Suppose that the axial velocity $u=u_{0}$ is a given constant, and the influences of the vertical plane motion and the parameters of the rolling motion are neglected; then we have

$$
\begin{aligned}
& \left(m-\frac{1}{2} \rho L^{3} Y_{\dot{v}}^{\prime}\right) \dot{v}+\left(-\frac{1}{2} \rho L^{4} Y_{\dot{r}}^{\prime}\right) \dot{r}=\left(\frac{1}{2} \rho L^{2} Y_{u v}^{\prime} u_{0}\right) v \\
& +\left(\frac{1}{2} \rho L^{3} Y_{u r}^{\prime} u_{0}-m u_{0}\right) r+\left(\frac{1}{2} \rho L^{2} Y_{\delta_{r}}^{\prime} u_{0}^{2}\right) \delta_{r} \\
& +\left[\frac{1}{2} \rho L^{4} Y_{r|r|}^{\prime} r|r|+\frac{1}{2} \rho L^{3} Y_{v|r|}^{\prime} v|r|+\frac{1}{2} \rho L^{2} Y_{v|v|}^{\prime} v|v|\right.
\end{aligned}
$$


TABLE 1: Notation used for AUVs.

\begin{tabular}{|c|c|c|c|c|}
\hline DOF & $\begin{array}{c}\text { Motion/rotation } \\
\text { description }\end{array}$ & Forces/moments & Linear/angular velocities & Positions/Euler angles \\
\hline 1 & $\begin{array}{l}\text { In } x \text {-direction } \\
\quad \text { (surge })\end{array}$ & $X$ & $u$ & $x$ \\
\hline 2 & $\begin{array}{c}\text { In } y \text {-direction } \\
\text { (sway) }\end{array}$ & $Y$ & $v$ & $y$ \\
\hline 3 & $\begin{array}{l}\text { In } z \text {-direction } \\
\text { (heave) }\end{array}$ & $Z$ & $w$ & $z$ \\
\hline 4 & $\begin{array}{l}\text { About } x \text {-axis } \\
\quad \text { (roll) }\end{array}$ & K & $p$ & $\phi$ \\
\hline 5 & $\begin{array}{l}\text { About } y \text {-axis } \\
\quad \text { (pitch) }\end{array}$ & $M$ & $q$ & $\theta$ \\
\hline 6 & $\begin{array}{l}\text { About } z \text {-axis } \\
\text { (yaw) }\end{array}$ & $N$ & $r$ & $\psi$ \\
\hline
\end{tabular}

$$
\begin{aligned}
& +(W-B) \cos \theta \sin \phi]+d \\
( & \left.-\frac{1}{2} \rho L^{4} N_{\dot{v}}^{\prime}\right) \dot{v}+\left(I_{z}-\frac{1}{2} \rho L^{5} N_{\dot{r}}^{\prime}\right) \dot{r}=\left(\frac{1}{2} \rho L^{3} N_{u v}^{\prime} u_{0}\right) v \\
& +\left(\frac{1}{2} \rho L^{4} N_{u r}^{\prime} u_{0}\right) r+\left(\frac{1}{2} \rho L^{3} N_{\delta_{r}}^{\prime} u_{0}^{2}\right) \delta_{r} \\
& +\left(\frac{1}{2} \rho L^{5} N_{r|r|}^{\prime} r|r|+\frac{1}{2} \rho L^{4} N_{|v| r}^{\prime}|v| r\right. \\
& \left.+\frac{1}{2} \rho L^{3} N_{v|v|}^{\prime} v|v|\right)+d \\
\dot{\psi} & =r .
\end{aligned}
$$

Define the heading instruction as a constant $\psi_{r}$, and $\dot{\psi}_{r}=$ 0 , heading error is $\psi_{e}(t)$, and then

$$
\begin{aligned}
& \psi_{e}=\psi_{r}-\psi . \\
& \dot{\psi}_{e}=\frac{d\left(\psi_{r}-\psi\right)}{d t}=-\dot{\psi}=-r .
\end{aligned}
$$

Considering the ocean current disturbances, an integral unit is introduced to eliminate the accumulated error

$$
\dot{\psi}_{I}=\psi_{e} \text {. }
$$

Define the state vector as $x=\left[\begin{array}{llll}v & r & \psi_{e} & \psi_{I}\end{array}\right]^{T} \in R^{4}$, the control vector $\delta_{r}(t)=u(t)$, and the dynamic model of the AUVs heading control system can be rewritten as follows:

$$
\dot{x}(t)=\left[\begin{array}{cccc}
a_{11} & a_{12} & 0 & 0 \\
a_{21} & a_{22} & 0 & 0 \\
0 & -1 & 0 & 0 \\
0 & 0 & 1 & 0
\end{array}\right]\left[\begin{array}{c}
v \\
r \\
\psi_{e} \\
\psi_{I}
\end{array}\right]+\left[\begin{array}{c}
b_{1} \\
b_{2} \\
0 \\
0
\end{array}\right] \delta_{r}+\left[\begin{array}{c}
d_{1} \\
d_{2} \\
0 \\
0
\end{array}\right] d
$$

$$
\begin{aligned}
& +\left[\begin{array}{c}
f_{1} \\
f_{2} \\
0 \\
0
\end{array}\right] \\
& =A x(t)+B u(t)+D d(t)+f(x, t), \\
x\left(t_{0}\right) & =x_{0},
\end{aligned}
$$

$$
\begin{aligned}
{\left[\begin{array}{ll}
a_{11} & a_{12} \\
a_{21} & a_{22}
\end{array}\right] } & =T^{-1} A_{1}, \\
{\left[\begin{array}{l}
b_{1} \\
b_{2}
\end{array}\right] } & =T^{-1} B_{1}, \\
{\left[\begin{array}{l}
d_{1} \\
d_{2}
\end{array}\right] } & =T^{-1}\left[\begin{array}{l}
1 \\
1
\end{array}\right], \\
{\left[\begin{array}{l}
f_{1} \\
f_{2}
\end{array}\right] } & =T^{-1}\left[\begin{array}{c}
\frac{1}{2} \rho L^{4} Y_{r|r|}^{\prime} r|r|+\frac{1}{2} \rho L^{3} Y_{v|r|}^{\prime} v|r|+\frac{1}{2} \rho L^{2} Y_{v|v|}^{\prime} v|v|+(W-B) \cos \theta \sin \phi \\
\frac{1}{2} \rho L^{5} N_{r|r|}^{\prime} r|r|+\frac{1}{2} \rho L^{4} N_{|v| r}^{\prime}|v| r+\frac{1}{2} \rho L^{3} N_{v|v|}^{\prime} v|v|
\end{array}\right],
\end{aligned}
$$




$$
\begin{aligned}
T & =\left[\begin{array}{cc}
m-\frac{1}{2} \rho L^{3} Y_{\dot{v}}^{\prime} & -\frac{1}{2} \rho L^{4} Y_{\dot{r}}^{\prime} \\
-\frac{1}{2} \rho L^{4} N_{\dot{v}}^{\prime} & I_{z}-\frac{1}{2} \rho L^{5} N_{\dot{r}}^{\prime}
\end{array}\right], \\
A_{1} & =\left[\begin{array}{cc}
\frac{1}{2} \rho L^{2} Y_{u v}^{\prime} u_{0} & \frac{1}{2} \rho L^{3} Y_{u r}^{\prime} u_{0}-m u_{0} \\
\frac{1}{2} \rho L^{3} N_{u v}^{\prime} u_{0} & \frac{1}{2} \rho L^{4} N_{u r}^{\prime} u_{0}
\end{array}\right], \\
B_{1} & =\left[\begin{array}{l}
\frac{1}{2} \rho L^{2} Y_{\delta_{r}}^{\prime} u_{0}^{2} \\
\frac{1}{2} \rho L^{3} N_{\delta_{r}}^{\prime} u_{0}^{2}
\end{array}\right] .
\end{aligned}
$$

$A, B$, and $D$ are defined in (6), $x_{0}$ is the initial state, and $f(x, t)$ is a limitary nonlinear vector, which includes errors and uncertainties.

Remark 1 . The limitary nonlinear vector $f(x, t)$ satisfies the following Lipschitz conditions:

$$
\begin{aligned}
&\|f(x, t)\| \leq \alpha\|x\|, \\
&\|f(x, t)-f(\widehat{x}, t)\| \leq \beta\|x-\widehat{x}\|, \\
& \forall x, \widehat{x} \in \Omega \subset R^{4},
\end{aligned}
$$

where $\alpha$ and $\beta$ are positive constants.

2.2. Disturbances Model of Wave Force. The external disturbances for AUVs are complex. In the near water surface, the wave force disturbances are the most important factor to the AUVs system. But ocean waves are always irregular. In present study, the irregular long storm waves are often simplified [31-33] as follows:

$$
\zeta_{a}(t)=\sum_{i=1}^{\infty} \zeta_{a i} \cos \left(k_{i} \xi \cos \mu+k_{i} \eta \sin \mu-\omega_{i} t+\varepsilon_{i}\right),
$$

where $k_{i}$ is the $i$ th component wave number, $\omega_{i}$ is the $i$ th component wave frequency, $\varepsilon_{i}$ is a random phase angle uniformly distributed within $0 \sim 2 \pi, \zeta_{a i}$ is the $i$ th component wave amplitude, $\zeta_{a i}=\sqrt{2 S\left(\omega_{i}\right) \Delta \omega}, S\left(\omega_{i}\right)$ is the $i$ th component ocean power spectrum density (PSD) function, and $\Delta \omega$ is the frequency discretization intervals of wave spectrum.

The wave force disturbances have been represented according to the Pierson-Moskowitz (PM) spectrum, written as

$$
S(\omega)=\frac{A}{\omega^{5}} \exp \left(\frac{-B}{\omega^{4}}\right),
$$

where $A=0.0081 g^{2}, B=3.12 /\left(H_{1 / 3}\right)^{2}$, where $g$ is the acceleration due to gravity, and $H_{1 / 3}$ is the significant wave height in meters.
Considering the stationary waves on the sea surface, let $\xi=0$ and $\eta=0$, and choose multiple regular wave superposition to get random waves, so the point long-crested waves are written as

$$
\begin{aligned}
\zeta_{a}(t) & =\sum_{i=1}^{N} \sqrt{2 S\left(\omega_{i}\right) \Delta \omega} \cos \left(\omega_{e i} t+\varepsilon_{i}\right) \\
& =\sum_{i=1}^{N} T_{i}\left(\omega_{i}\right) \cos \theta_{i},
\end{aligned}
$$

where $N$ is the number of superimposed waves, $\omega_{e i}=\omega_{i}-$ $\left(\omega_{i}{ }^{2} u_{0} / g\right) \cos \beta$ is encounter angle frequency, and $\beta$ is the encounter wave angle.

We construct a system model to describe the irregular wave forces for the AUV in two-dimensional horizontal plane.

Define $v_{i}=T_{i}\left(\omega_{i}\right) \cos \left(\theta_{i}\right)$ as the horizontal velocity of water particle orbital motion.

Let $v(t)=\left[\begin{array}{lll}v_{1}(t) & \cdots & v_{N}(t)\end{array}\right]^{T}$; taking the derivative of $v(t)$, we have

$$
\ddot{v}_{i}=-\omega_{i}^{2} v_{i}=-\Omega v,
$$

where $\Omega=\operatorname{diag}\left\{\omega_{1}^{2}, \omega_{2}^{2}, \ldots, \omega_{N}^{2}\right\}$.

Define $w(t)=\left[v(t)^{T}, \dot{v}(t)^{T}\right]^{T}$; then

$$
\begin{aligned}
& \dot{w}(t)=\left[\begin{array}{cc}
0 & I \\
-\Omega & 0
\end{array}\right] w(t) \triangleq F w(t) \\
& v(t)=\left[\begin{array}{ll}
I & 0
\end{array}\right] w(t)
\end{aligned}
$$

where $I$ is $N$ dimensional unit matrix and 0 is $N$ dimensional zero matrix. 
According to the linear wave theory, the wave force disturbances for the AUV system are as follows:

$$
\begin{aligned}
d(t) & =\sum_{i=1}^{N} T_{i}\left(\omega_{i}\right) v_{i}(t) \\
& =\left[\begin{array}{llll}
T_{1}\left(\omega_{1}\right) & \cdots & T_{N}\left(\omega_{N}\right)
\end{array}\right]\left[\begin{array}{llll}
v_{1}(t) & \cdots & v_{N}(t)
\end{array}\right]^{T} \\
& =\left[\begin{array}{llll}
T_{1}\left(\omega_{1}\right) & \cdots & T_{N}\left(\omega_{N}\right)
\end{array}\right]\left[\begin{array}{ll}
I & 0
\end{array}\right] w(t) \triangleq H w(t) .
\end{aligned}
$$

So the total wave force disturbances for AUVs can be described by the following system:

$$
\begin{aligned}
& \dot{w}(t)=F w(t), \\
& d(t)=H w(t),
\end{aligned}
$$

where $F$ and $H$ are real constant matrices of appropriate dimensions.

\section{Controller Design}

According to the dynamic model of the AUVs heading control system (6), we select the following average quadratic performance index:

$$
J=\lim _{T \rightarrow \infty} \frac{1}{T} \int_{t_{0}}^{T}\left[x^{T}(t) Q x(t)+u^{T}(t) R u(t)\right] d t,
$$

where $Q$ is the weighting matrix for states, symmetric positive semidefinite, and $R$ is the weighting matrix for the control inputs, symmetric positive definite. $Q$ and $R$ are properly selected to shape the response characteristics in the closedloop AUVs heading control system (6).

The optimal control problem is to search for a control law $u^{*}(t)$ for system (6), which makes the value of the average quadratic performance index (16) minimum.

Applying the maximum principle of the optimal control problem in (6) and (16), the optimal control law can be written as

$$
u^{*}(t)=-R^{-1} B^{T} \lambda(t),
$$

where $\lambda(t)$ is the solution to the following TPBV problem:

$$
\begin{aligned}
-\dot{\lambda}(t) & =Q x(t)+A^{T} \lambda(t)+f_{x}^{T}(x, t) \lambda(t), \\
\dot{x}(t) & =A x(t)-S \lambda(t)+f(x, t)+D d(t), \\
x\left(t_{0}\right) & =x_{0}, \\
\lambda(\infty) & =0,
\end{aligned}
$$

$$
t>t_{0}
$$

which is the optimality necessary condition, where $S=$ $B R^{-1} B^{T}$ and $f_{x}^{T}(x, t)=\partial f^{T}(x, t) / \partial x$.

For the AUVs heading control system (6) and wave force system (15) with the average quadratic performance index (16), we now state the following theorem.
Theorem 2. Consider the optimal control problem described by systems (6) and (15) with the average quadratic performance index (16). Then the ODRC law $u^{*}(t)$ is existent and unique, and its form is as follows:

$$
u^{*}(t)=-R^{-1} B^{T}\left[P x(t)+\bar{P} w(t)+\lim _{k \rightarrow \infty} g^{(k)}(t)\right],
$$

where $P$ is the unique positive-definite solution of the following Riccati matrix equation:

$$
A^{T} P+P A-P S P+Q=0 .
$$

$\bar{P}$ is the unique solution of the following Sylvester matrix equation:

$$
(A-S P)^{T} \bar{P}+\bar{P} F=-P D H .
$$

Proof. Let

$$
\lambda(t)=P x(t)+P_{1} d(t)+g(t),
$$

where $x(t), d(t)$, and $g(t)$ are the state vector, the wave force disturbances, and the adjoint vector, respectively.

It is well known that $P$ is the unique positive-definite solution of Riccati matrix equation (20). Substituting $P$ into (21), then $\bar{P}$ can be solved uniquely; here $\bar{P}=P_{1} H$. When $P$ and $\bar{P}$ are got uniquely, therefore, we can obtain the ODRC law uniquely as follows:

$$
u^{*}(t)=-R^{-1} B^{T}[P x(t)+\bar{P} w(t)+g(t)],
$$

where $g(t)$ and $x(t)$ are the solutions of the following equations:

$$
\begin{aligned}
\dot{g}(t)= & \left(P S-A^{T}\right) g(t)-P f(x, t) \\
& -f_{x}^{T}(x, t)[P x(t)+\bar{P} w(t)+g(t)], \\
\dot{x}(t)= & (A-S P) x(t)+(D H-S \bar{P}) w(t)-S g(t) \\
& +f(x, t), \\
g(\infty)= & 0, \\
x\left(t_{0}\right)= & x_{0},
\end{aligned}
$$

and $w(t)$ is the solution of the wave forces system (15).

By using the successive approximation approach $[26,27$, $31,34]$, we construct the adjoint vector sequence

$$
\begin{aligned}
& g^{(0)}(t) \equiv 0, \\
& \dot{g}^{(k)}(t) \\
& =\left(P S-A^{T}\right) g^{(k)}(t)-P f\left(x^{(k-1)}, t\right) \\
& \quad-f_{x}^{T}\left(x^{(k-1)}, t\right)\left[P x^{(k-1)}(t)+\bar{P} w(t)+g^{(k-1)}(t)\right], \\
& \lim _{T \rightarrow \infty} g^{(k)}(T)=0,
\end{aligned}
$$

$$
k=1,2, \ldots
$$


and the state equation sequence

$$
\begin{aligned}
x^{(0)}(t) \equiv & 0, \\
\dot{x}^{(k)}(t)= & (A-S P) x^{(k)}(t)+(D H-S \bar{P}) w(t) \\
& \quad-S g^{(k)}(t)+f\left(x^{(k-1)}, t\right), \\
x^{(k)}\left(t_{0}\right)= & x_{0}, \quad k=1,2, \ldots
\end{aligned}
$$

It can be proved that the adjoint vector solution sequence $\left\{g^{(k)}(t)\right\}$ in (25) uniformly converges to $g(t)$, and the state vector solution sequence $\left\{x^{(k)}(t)\right\}$ in (26) uniformly converges to $x(t)[26,27,31,34]$.

When $k \rightarrow \infty$, the limits of $\left\{x^{(k)}(t)\right\}$ and $\left\{g^{(k)}(t)\right\}$ become the optimal state vector $x^{*}(t)$ and the optimal adjoint vector $g(t)$.

Define $g^{(\infty)}(t)=\lim _{k \rightarrow \infty} g^{(k)}(t)$; then the ODRC law is rewritten as (19). This completes the proof.

Remark 3. It is usually impossible to obtain the exact adjoint vector $g^{(\infty)}(t)$ when designing the ODRC law in practice. In many cases, it may be better to choose an $g^{(N)}(t)$ as the approximation of $g^{(\infty)}(t)$ where $N$ depends on a concrete error coefficient $\varepsilon>0$. The $N$ th-order ODRC law is as follows:

$$
u_{N}(t)=-R^{-1} B^{T}\left[P x(t)+\bar{P} w(t)+g^{(N)}(t)\right] .
$$

Remark 4. The Nth-order ODRC law consists of a feedback term $P x(t)$, a feedforward disturbances rejection term $\bar{P} w(t)$, and a nonlinear compensatory term $g^{(N)}(t)$.

Remark 5. The wave disturbances are difficult to be measured and obtained, and $w(t)$ is the state vectors of system (15); the ODRC law is physically unrealizable in practice. So, we construct a Luenberger disturbances observer to make it realizable as follows:

$$
\dot{\widehat{w}}(t)=(F-L H) \widehat{w}(t)+L d(t)
$$

where $\widehat{w}(t)$ is the observation value of $w(t)$ and $L$ is the observer matrix of appropriate dimensions. We can choose the appropriate dimensions matrix $L$, and the eigenvalues of the matrix $(F-L H)$ have negative real parts. Then the $N$ thorder ODRC law is as follows:

$$
\begin{aligned}
& u_{N}(t)=-R^{-1} B^{T}\left[P x(t)+\bar{P} \widehat{w}(t)+g^{(N)}(t)\right], \\
& \dot{\widehat{w}}(t)=(F-L H) \widehat{w}(t)+L d(t) .
\end{aligned}
$$

\section{Example and Simulation}

4.1. Simulation Model and Parameters for AUVs and Wave Force Disturbances. In this section, a simulation study is carried out using a typical AUV model [35] to demonstrate the performance of the proposed approach. The hydrodynamic coefficients of an AUV are shown in Table 2.
TABLE 2: Hydrodynamic coefficients of the AUV.

\begin{tabular}{lccc}
\hline Name & Symbol & Numerical & Unit \\
\hline Quality & $m$ & 6783.1 & $\mathrm{~kg}$ \\
Length & $L$ & 5.720 & $\mathrm{~m}$ \\
Sea-water density & $\rho$ & 1.025 & $\mathrm{~kg} / \mathrm{m}^{3}$ \\
& $Y^{\prime}{ }_{\dot{v}}$ & -39.854 & \\
Dimensionless & $Y^{\prime}{ }_{\dot{r}}$ & 12.567 & \\
coefficient of sway & $Y^{\prime}{ }_{v|v|}$ & -25.704 & \\
force & $Y^{\prime}{ }_{r|r|}$ & 9.981 & \\
& $Y^{\prime}{ }^{u v}$ & -41.858 & \\
& $Y^{\prime}{ }^{\prime}{ }^{\prime}$ & 10.435 & \\
& $Y^{\prime}{ }_{\delta r}$ & 18.509 & \\
& $N^{\prime}{ }_{\dot{v}}$ & -3.872 & $10^{-3}$ \\
Dimensionless & $N^{\prime}{ }_{\dot{r}}$ & -2.105 & \\
coefficient of yaw & $N^{\prime}{ }_{v|v|}$ & 17.480 & \\
moment & $N^{\prime}{ }_{r|r|}$ & -6.463 & \\
& $N^{\prime}{ }_{u v}$ & -2.878 & \\
& $N^{\prime}{ }_{u r}$ & -9.436 & \\
\hline & $N^{\prime}{ }_{\delta r}$ & -9.754 & \\
\hline
\end{tabular}

Assuming that the axial velocity is $u_{0}=2 \mathrm{~m} / \mathrm{s}$, the significant wave height is $H_{1 / 3}=4 \mathrm{~m}$, and encounter angle frequency of wave is $0.6320 \mathrm{rad} / \mathrm{s}$ and $1.0470 \mathrm{rad} / \mathrm{s}$, then the PSD of the wave height is shown in Figure 2.

And the wave force can be calculated from (11), which is shown in Figure 3.

The matrices and vector of the AUVs heading control system (6) are as follows:

$$
A=\left[\begin{array}{cccc}
-0.1966 & -1.1472 & 0 & 0 \\
-0.0780 & -0.6558 & 0 & 0 \\
0 & -1 & 0 & 0 \\
0 & 0 & 1 & 0
\end{array}\right]
$$$$
B=\left[\begin{array}{c}
0.1962 \\
0.2165 \\
0 \\
0
\end{array}\right]
$$$$
D=\left[\begin{array}{c}
0.0002 \\
0.0273 \\
0 \\
0
\end{array}\right]
$$

$$
f(x, t)=\left[\begin{array}{c}
-1.6977 x_{1}{ }^{2}+2.4396 x_{2}{ }^{2}-2.6767 x_{1} x_{2} \\
0.0458 x_{1}{ }^{2}-0.5541 x_{2}{ }^{2}-0.0365 x_{1} x_{2} \\
0 \\
0
\end{array}\right] \text {. }
$$


TABLE 3: $J_{N}$ and $\Delta J$ at different iteration times.

\begin{tabular}{cccccc}
\hline$k$ & 1 & 2 & 3 & 4 & 5 \\
\hline$J_{N}$ & 15.2455 & 11.3397 & 10.1086 & 9.7592 & 9.7452 \\
$\Delta J$ & $/$ & 0.3444 & 0.1218 & 0.0358 & 0.0014 \\
\hline
\end{tabular}

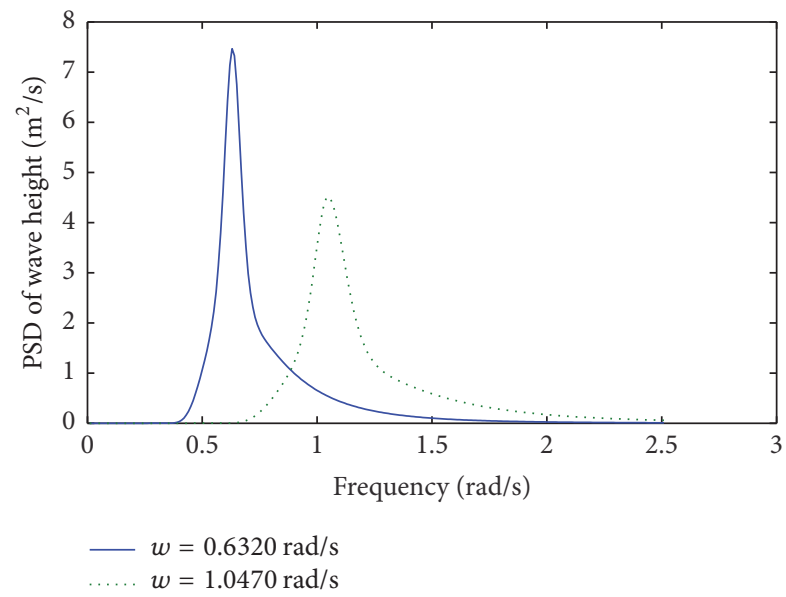

FIGURE 2: PSD of wave height.

The parameters for the average quadratic performance index (16) are chosen as

$$
\begin{aligned}
Q & =\left[\begin{array}{llll}
1 & 0 & 0 & 0 \\
0 & 1 & 0 & 0 \\
0 & 0 & 1 & 0 \\
0 & 0 & 0 & 1
\end{array}\right], \\
R & =5 .
\end{aligned}
$$

By solving (20) and (21), we obtain

$$
\begin{aligned}
& P=\left[\begin{array}{cccc}
6.5444 & -13.9363 & 3.1885 & 0.2422 \\
-13.9363 & 79.6598 & -56.8132 & -10.5478 \\
3.1885 & -56.8132 & 56.9366 & 13.1293 \\
0.2422 & -10.5478 & 13.1293 & 5.2210
\end{array}\right], \\
& \bar{P}=\left[\begin{array}{cccc}
-1.2713 & -0.6152 & -11.2055 & -5.4904 \\
19.5865 & 9.1021 & 57.4458 & 30.7596 \\
-19.6954 & -9.5852 & -34.9479 & -20.9735 \\
-4.5788 & -2.4965 & -4.2104 & -3.4488
\end{array}\right] .
\end{aligned}
$$

4.2. Simulation Results and Analysis. Based on the AUV model and parameters, the state vector is considered as $x=\left[\begin{array}{llll}v & r & \psi_{e} & \psi_{I}\end{array}\right]^{T}$; the simulation curves $x(t)$ and $u(t)$ at different iteration times are shown in Figures 4-8.

The average quadratic performance index values $J_{N}$ and errors $\Delta J=\left|\left(J_{N-1}-J_{N}\right) / J_{N}\right|<\varepsilon$, at different iteration times, are listed in Table 3 , and $\varepsilon$ is the control precision.

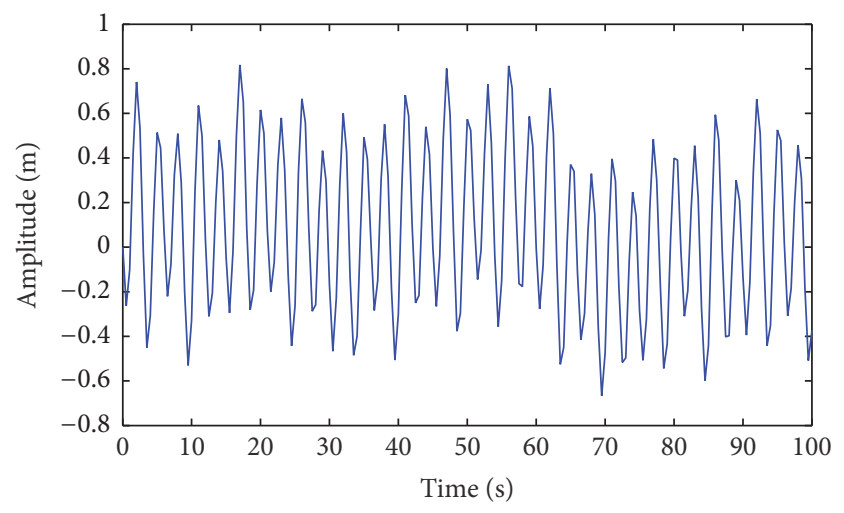

FIGURE 3: Numerical simulation of the random wave force.

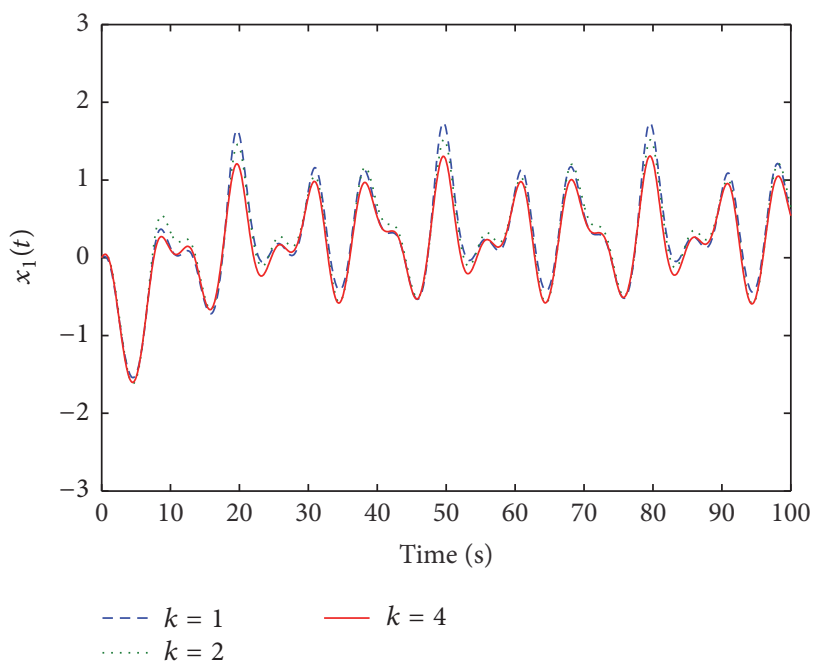

FIGURE 4: The simulation curves of $x_{1}(t)$.

From Table 3, it can be seen clearly that the average quadratic performance index values decrease as iteration times increase and tend to a deterministic optimal value $J^{*}$ ultimately. If we choose the control precision $\varepsilon=0.05$, then the relative error of the average quadratic performance index values satisfies $\left|\left(J_{3}-J_{4}\right) / J_{4}\right|<\varepsilon$. It indicates that the 4 th ODRC law $u_{4}(t)$ is very close to the optimal control law $u^{*}(t)$. It is obvious that the proposed approach requires a few iterations to get the approximate ODRC law. And it is more robust about current and wave disturbances. Simulation results show that the proposed approach applied to the AUVs is effective.

\section{Conclusion}

In this paper, the disturbances rejection control problem for the AUVs heading control system has been considered, and an ODRC approach has been designed. For the design, nonlinearities in the AUVs system are retained, and disturbances for the AUVs system are considered. By using a successive approximation approach, an ODRC law is proposed based on the quadratic optimal control theory, 


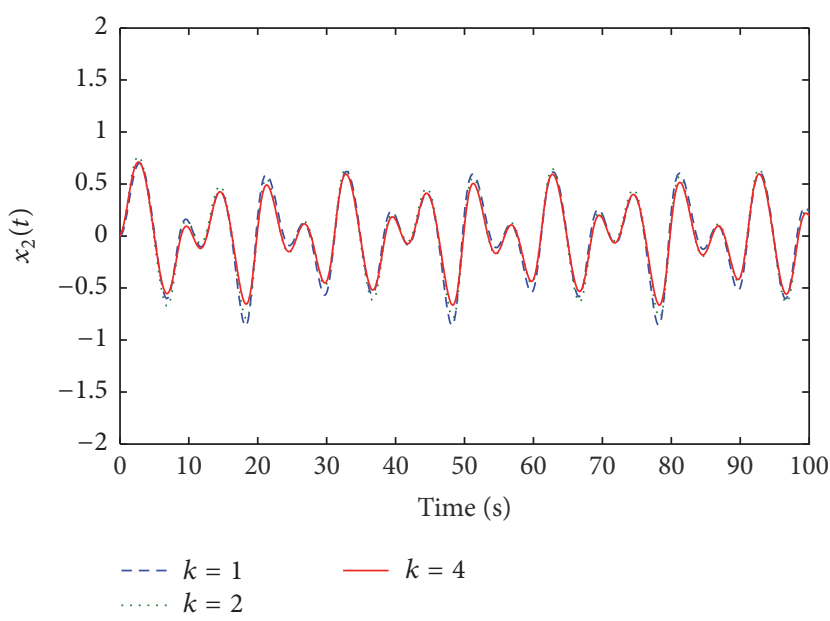

FIgURE 5: The simulation curves of $x_{2}(t)$.

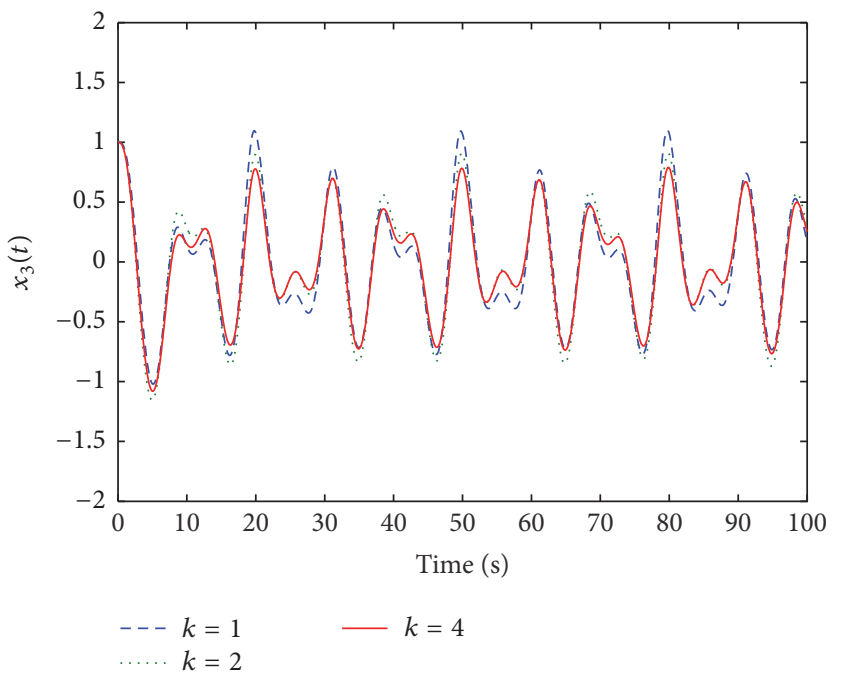

Figure 6: The simulation curves of $x_{3}(t)$.

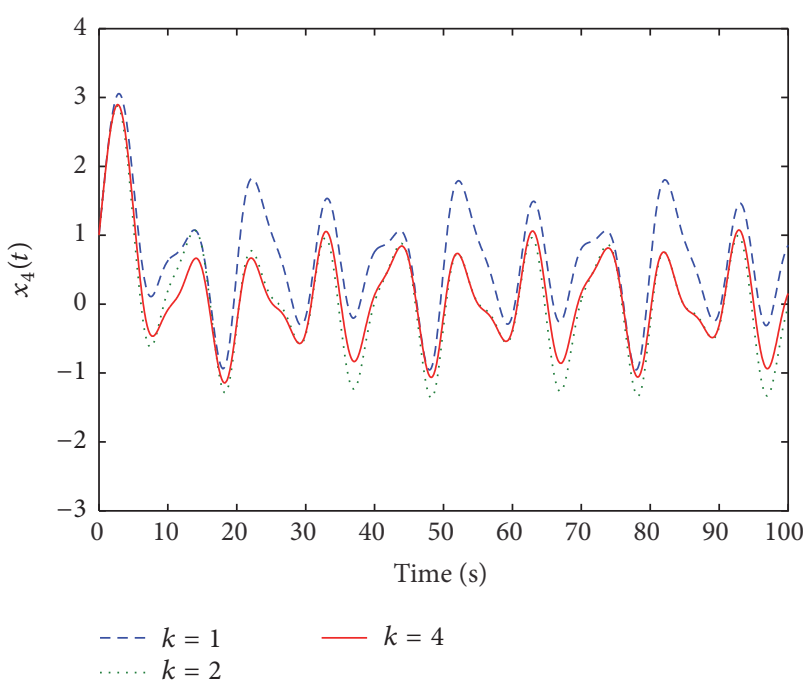

Figure 7: The simulation curves of $x_{4}(t)$.

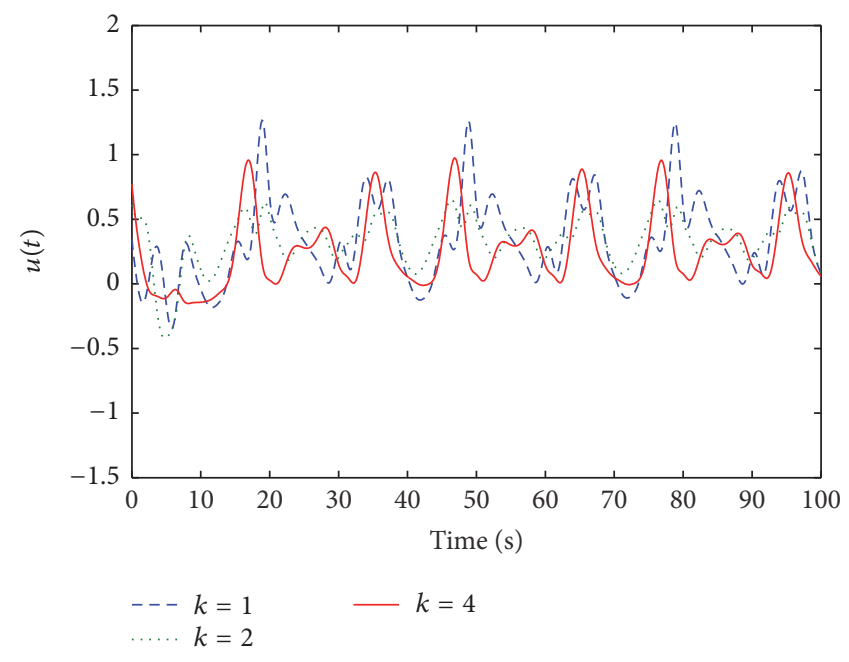

FIgURE 8: The simulation curves of $u(t)$.

which consists of the optimal feedback item, the feedforward disturbances rejection item, and the nonlinear compensatory item. Finally, the effectiveness of the proposed approach has been illustrated by an AUV model.

\section{Conflicts of Interest}

The authors declare that they have no conflicts of interest.

\section{Acknowledgments}

This study was supported in part by the National Natural Science Foundation of China (61673357, 41276085, and 61572448) and by the Natural Science Excellence Foundation of Shandong Province (ZR2015FM004 and ZR2014JL043).

\section{References}

[1] R. B. Wynn, V. A. I. Huvenne, T. P. Le Bas et al., "Autonomous Underwater Vehicles (AUVs): their past, present and future contributions to the advancement of marine geoscience," Marine Geology, vol. 352, pp. 451-468, 2014.

[2] G. Bartolini and A. Pisano, "Black-box position and attitude tracking for underwater vehicles by second-order slidingmode technique," International Journal of Robust and Nonlinear Control, vol. 20, no. 14, pp. 1594-1609, 2010.

[3] L. Wang, H.-M. Jia, L.-J. Zhang, and H.-B. Wang, "Horizontal tracking control for AUV based on nonlinear sliding mode," in Proceedings of the IEEE International Conference on Information and Automation (ICIA '12), vol. 1, pp. 460-463, 2012.

[4] Z.-P. Yan, D. Wu, J.-J. Zhou, and X. Zhang, "Application of extension integral variable structure control method on simulation of AUV heading control system," in Proceedings of the 30th Chinese Control Conference, vol. 7, pp. 2617-2621, July 2011.

[5] J. Ghommam and M. Saad, "Backstepping-based cooperative and adaptive tracking control design for a group of underactuated AUVs in horizontal plan," International Journal of Control, vol. 87, no. 5, pp. 1076-1093, 2014. 
[6] L. Lapierre and B. Jouvencel, "Robust nonlinear path-following control of an AUV," IEEE Journal of Oceanic Engineering, vol. 33, no. 2, pp. 89-102, 2008.

[7] L. Lapierre and D. Soetanto, "Nonlinear path-following control of an AUV," Ocean Engineering, vol. 34, no. 11-12, pp. 1734-1744, 2007.

[8] J. Petrich and D. J. Stilwell, "Robust control for an autonomous underwater vehicle that suppresses pitch and yaw coupling," Ocean Engineering, vol. 38, no. 1, pp. 197-204, 2011.

[9] L. Moreira and C. G. Soares, " $H_{2}$ and $H_{\infty}$ designs for diving and course control of an Autonomous underwater vehicle in presence of waves," Journal of Oceanic Engineering, vol. 33, no. 2, pp. 69-88, 2008.

[10] B. K. Sahu and B. Subudhi, "Adaptive tracking control of an autonomous underwater vehicle," International Journal of Automation and Computing, vol. 11, no. 3, pp. 299-307, 2014.

[11] B. B. Miao, T. S. Li, and W. L. Luo, "A DSC and MLP based robust adaptive NN tracking control for underwater vehicle," Neurocomputing, vol. 111, pp. 184-189, 2013.

[12] L.-J. Zhang, X. Qi, and Y.-J. Pang, "Adaptive output feedback control based on DRFNN for AUV," Ocean Engineering, vol. 36, no. 9-10, pp. 716-722, 2009.

[13] B. Geranmehr and S. R. Nekoo, "Nonlinear suboptimal control of fully coupled non-affine six-DOF autonomous underwater vehicle using the state-dependent Riccati equation," Ocean Engineering, vol. 96, pp. 248-257, 2015.

[14] D. W. Kim, "Tracking of REMUS autonomous underwater vehicles with actuator saturations," Automatica, vol. 58, pp. 1521, 2015.

[15] F. Repoulias and E. Papadopoulos, "Planar trajectory planning and tracking control design for underactuated AUVs," Ocean Engineering, vol. 34, no. 11-12, pp. 1650-1667, 2007.

[16] S.-W. Shi, W.-S. Yan, J. Gao, and W.-B. Li, "Path-following control of an AUV in the horizontal plane with constant ocean currents," Acta Armamentarii, vol. 31, no. 3, pp. 375-379, 2010.

[17] X.-Q. Cheng, J.-Y. Qu, Z.-P. Yan, and X.-Q. Bian, " $\mathrm{H}_{\infty}$ robust fault-tolerant controller design for an autonomous underwater vehicle's navigation control system," Journal of Marine Science and Application, vol. 9, no. 1, pp. 87-92, 2010.

[18] S. Liu, D. Wang, and E. Poh, "Non-linear output feedback tracking control for AUVs in shallow wave disturbance condition," International Journal of Control, vol. 81, no. 11, pp. 1806-1823, 2008.

[19] A. P. Aguiar and A. M. Pascoal, "Dynamic positioning and waypoint tracking of underactuated AUVs in the presence of ocean currents," International Journal of Control, vol. 80, no. 7, pp. 1092-1108, 2007.

[20] F.-D. Gao, C.-Y. Pan, Y.-Y. Han, and X. Zhang, "Nonlinear trajectory tracking control of a new autonomous underwater vehicle in complex sea conditions," Journal of Central South University, vol. 19, no. 7, pp. 1859-1868, 2012.

[21] L.-J. Zhang, X. Qi, Y.-J. Pang, and D.-P. Jiang, "Adaptive output feedback control for trajectory tracking of AUV in wave disturbance condition," International Journal of Wavelets, Multiresolution and Information Processing, vol. 11, no. 3, pp. 1$15,2013$.

[22] H. Joe, M. Kim, and S.-C. Yu, "Second-order sliding-mode controller for autonomous underwater vehicle in the presence of unknown disturbances," Nonlinear Dynamics, vol. 78, no. 1, pp. 183-196, 2014.
[23] H.-G. Zhang, Q.-L. Wei, and Y.-H. Luo, "A novel infinite-time optimal tracking control scheme for a class of discrete-time nonlinear systems via the greedy HDP iteration algorithm," IEEE Transactions on Systems, Man, and Cybernetics, Part B: Cybernetics, vol. 38, no. 4, pp. 937-942, 2008.

[24] Q.-L. Wei, H.-G. Zhang, D.-R. Liu, and Y. Zhao, "An optimal control scheme for a class of discrete-time nonlinear systems with time delays using adaptive dynamic programming," Acta Automatica Sinica, vol. 36, no. 1, pp. 121-129, 2010.

[25] Q.-X. Qu, Y.-H. Luo, and H.-G. Zhang, "Robust approximate optimal tracking control of time-varying trajectory for nonlinear affine systems," Control Theory \& Applications, vol. 33, no. 1, pp. 77-84, 2016.

[26] G.-Y. Tang, "Suboptimal control for nonlinear systems: a successive approximation approach," Systems and Control Letters, vol. 54, no. 5, pp. 429-434, 2005.

[27] G.-Y. Tang and D.-X. Gao, "Approximation design of optimal controllers for nonlinear systems with sinusoidal disturbances," Nonlinear Analysis: Theory, Methods \& Applications, vol. 66, no. 2, pp. 403-414, 2007.

[28] T. I. Fossen, Guidance and Control of Ocean Vehicles, John Wiley \& Sons, New York, NY, USA, 1994.

[29] T. Prestero, Verification of a Six-Degree of Freedom Simulation Model for the REMUS Autonomous Underwater Vehicles, MIT, 2001.

[30] O.-E. Fjellstad and T. I. Fossen, "Position and attitude tracking of AUV's: a quaternion feedback approach," IEEE Journal of Oceanic Engineering, vol. 19, no. 4, pp. 512-518, 1994.

[31] Q. Yang, H. Su, and G.-Y. Tang, "Approximate optimal tracking control for near-surface AUVs with wave disturbances," Journal of Ocean University of China, vol. 15, no. 5, pp. 789-798, 2016.

[32] H. Ma, G.-Y. Tang, and W. Hu, "Feedforward and feedback optimal control with memory for offshore platforms under irregular wave forces," Journal of Sound and Vibration, vol. 328, no. 4-5, pp. 369-381, 2009.

[33] B.-L. Zhang and Q.-L. Han, "Network-based modelling and active control for offshore steel jacket platform with TMD mechanisms," Journal of Sound and Vibration, vol. 333, pp. 6796-6814, 2014.

[34] S.-Y. Han, D. Wang, Y.-H. Chen, G.-Y. Tang, and X.-X. Yang, "Optimal tracking control for discrete-time systems with multiple input delays under sinusoidal disturbances," International Journal of Control, Automation and Systems, vol. 13, no. 2, pp. 292-301, 2015.

[35] X.-Q. Cheng, Research on the Robust Heading Control for $A U V$ Based on Feedback Linearization in Near Surface, Harbin Engineering University, Harbin, China, 2008. 


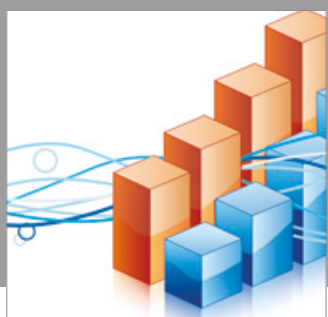

Advances in

Operations Research

vatersals

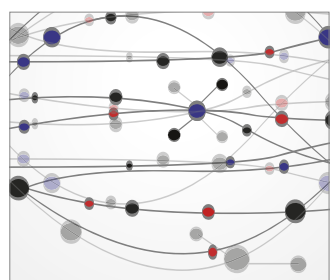

\section{The Scientific} World Journal
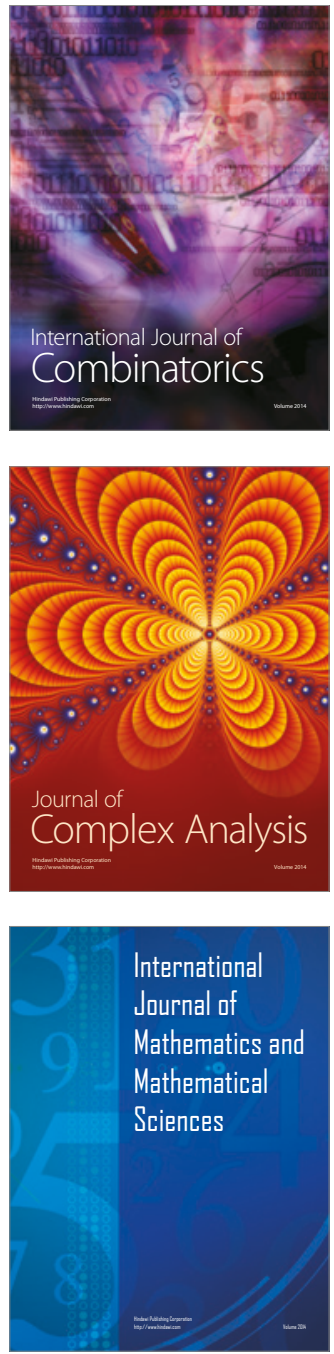
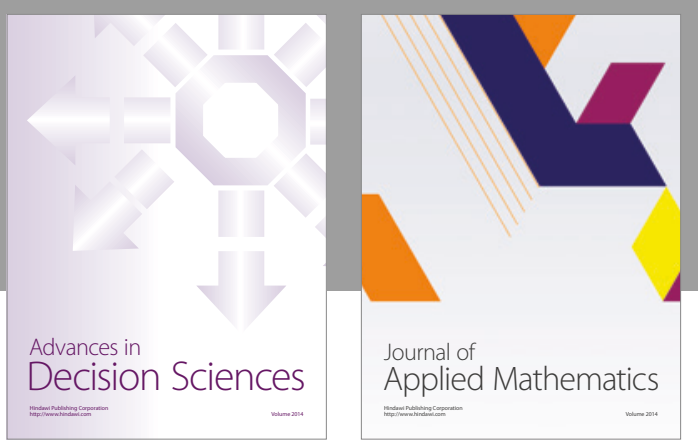

Algebra

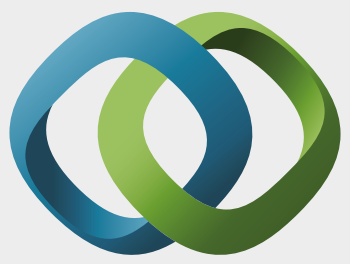

\section{Hindawi}

Submit your manuscripts at

https://www.hindawi.com
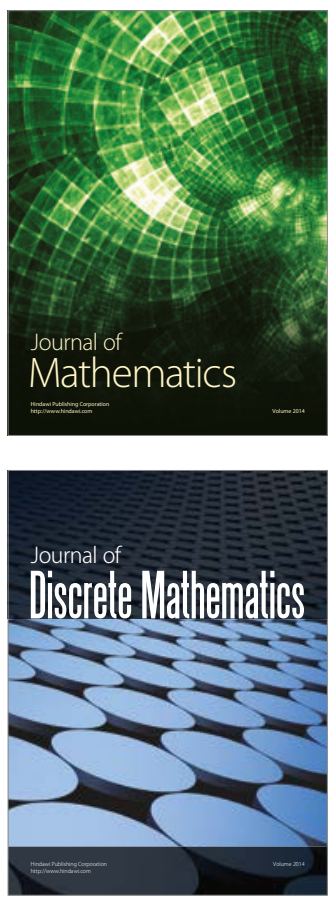

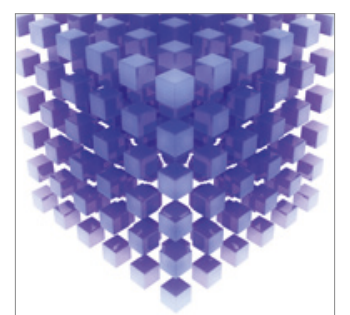

Mathematical Problems in Engineering
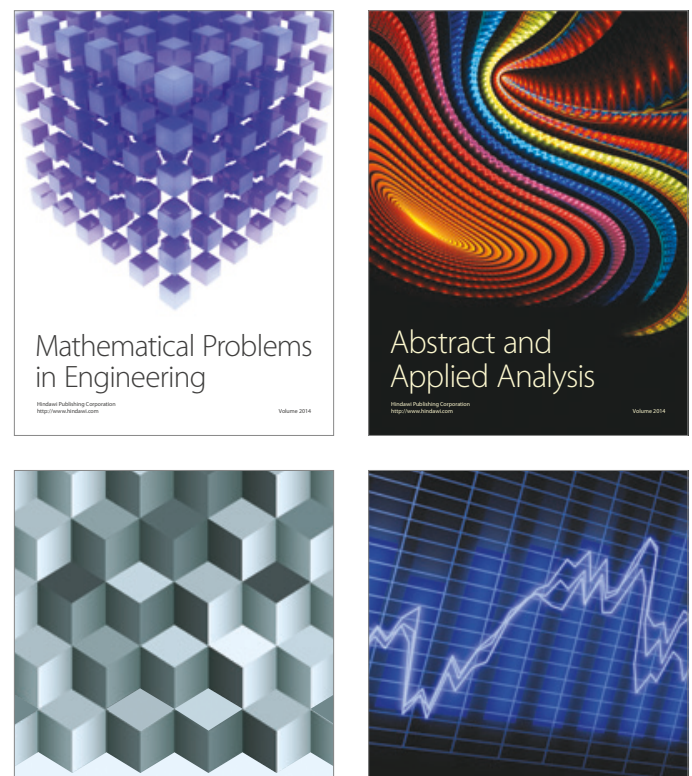

Journal of

Function Spaces

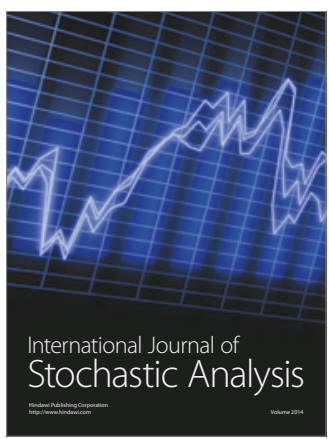

Probability and Statistics
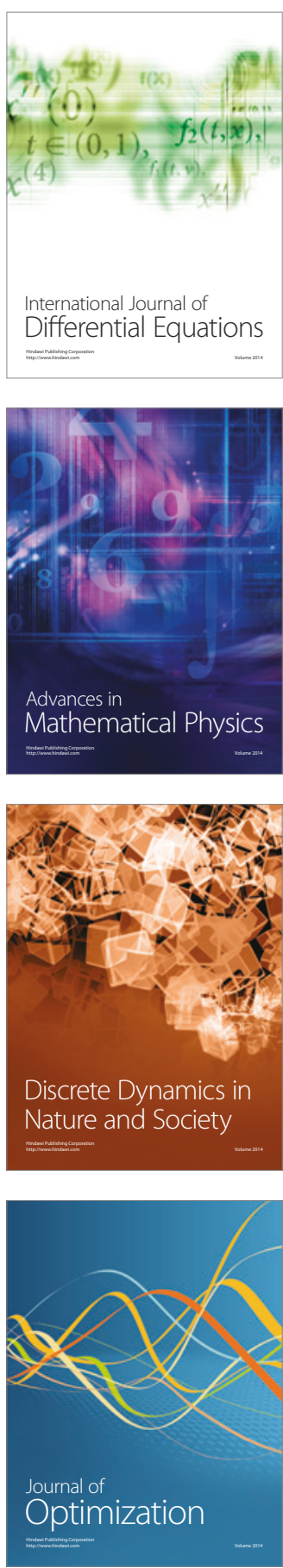